Journal of

Neurophysiology and Neurological Disorders

\title{
Case Report: Occlusion of the Artery of Percheron Leading to a Bilateral Thalamic Infarct in a Patient with Atrial Fibrillation
}

\section{Sohal R MD ${ }^{1, *}$, Sah B MD ${ }^{2}$}

${ }^{1}$ Internal Medicine, SUNY Upstate Medical University, 750 E Adams St, Syracuse, NY 13210.

${ }^{2}$ Assistant Professor Medical ICU director, Pulmonary \& Critical Care Medicine SUNY Upstate Medical University Syracuse, 90 Presidential Plaza, Pulmonology Syracuse, NY 13202.

${ }^{*}$ Corresponding author: Sohal R MD, Internal Medicine, SUNY Upstate Medical University, 750 E Adams St, Syracuse, NY 13210; Tel: 315-802-1291; E-mail: sohalr@upstate.edu/rjeetsohal@gmail.com

Received Date: May 24, 2019 Accepted Date: June 22, 2019 Published Date: June 25, 2019

Citation: Sohal R (2019) Case Report: Occlusion of the Artery of Percheron Leading to a Bilateral Thalamic Infarct in a Patient with Atrial Fibrillation. J Neurophysiol Neurol Disord 5: 1-3.

\begin{abstract}
An infarct in the artery of Percheron (AOP) is a rare cause of bilateral thalamic stroke in a patient that presents with comatosis [1] Although rare, in the absence of other more compelling and common causes stemming from metabolic derangements as acute kidney injury, infection, intoxication with hypercapnic respiratory failure; stroke should be high on the differential.
\end{abstract}

Although initial imaging on CT may not sure show a stroke, this differential should be pursued through further imaging with MRI; particularly in the case in which a patient presents with the triad of a sudden change in mentation, hypersomnolence, and vertical gaze palsy in a patient with known cardioembolic risk factors [1].

This case reports such a patient with decreased consciousness and NIH stroke score of 28 with an admission VBG showing mild hypercapnic respiratory failure and positive for coronavirus. Initially as the etiology of the behavioral change was unclear, the patient was started on broad-spectrum antibiotics; lactic acid was drawn which was elevated at 2.4 and patient was placed on BIPAP with subsequent improvement on ABG; but without improvement in mentation. The patient was unarousable despite several days of antibiotics, normal TSH, an unremarkable CBC, CMP, UA, and CXR. CT head was unrevealing. Patient remained unarousable.

MRI was then performed which showed stroke in the artery of Percheron. Due to the rarity of this anatomic variant, an AOP infarct is frequently missed; and this case highlights the importance of maintaining high suspicion of an AOP infract in a patient who presents with a triad of hypersolmnelsce, poor memory, and ophthalmologic sign as vertical gazy palsy. This is especially true in a patient with known risks factors for cardioembolic disease.

C2019 The Authors. Published by the JScholar under the terms of the Creative Commons Attribution License http://creativecommons.org/licenses/ by/3.0/, which permits unrestricted use, provided the original author and source are credited. 


\section{Admission course}

This reports a case of a male in his 80 s with sensorineural deafness, left eye enucleation advanced Alzheimer's dementia, atrial fibrillation not on anticoagulation and hypothyroidism residing at the nursing home who was brought to the ED for altered mental status. CHADS-VASc score was 7. Prior to admission the patient was not on oral anticoagulation; although he was on amiodarone for atrial fibrillation. On admission infectious workup was ordered which was negative. TSH level was normal. Patient was DNR/DNI and consequently, his oral medications could not be given. He remained in sinus rhythm while on telemetry patient which as attributed to the long halflife of the amiodarone. CT head non-contrast was performed on admission which did not show acute intracranial pathology. He was given levothyroxine IV. Blood cultures, sputum cultures were negative. CXR did not show consolidation. The initial working diagnosis was presumed to be acute hypercapnic respiratory failure secondary to aspiration pneumonia/ coronavirus positive. He was placed on BIPAP, ABG improved; but he remained encephalopathic. He was then taken off BIPAP and maintained on 2L of oxygen by nasal-cannula. No obvious infectious or metabolic etiology was found which could explain the lack of arousability. MRI brain was ordered for further evaluation which subsequently showed the bilateral thalamic stroke. Since there was no improvement in his mental status after 5 days, the family wished to transition his care to comfort care.

\section{Etiology}

Artery of Percheron is named after Gerard Percheron in 1973. He was a neuropathologist who first described this pattern of vascular supply as a single arterial trunk that divides into two to supply both the left and right paramedian thalamic nuclei. It is a rare anatomic variant of thalamic supply in the population [2]. It is a part of the posterior circulation and is a branch of the posterior cerebral artery from P1. Posterior circulation strokes are uncommon, and a bilateral thalamic stroke is an even more uncommon presentation of a posterior circulation stroke [2].

The important point is that the AOP infarct results in a characteristic pattern which is important to recognize. A case series was conducted by Lazarro et al which analyzed 37 patients retrospectively from across hospitals in the US who had AOP infarct based on the inclusion criteria of the presence of infarct on MRI of hypoattenuation in the area of the rostral pons on CT head [3]. The conclusion was that there were 4 types of AOP strokes; two with the involvement of the rostral midbrain; and two without [3].

The classical imaging finding on the MRI is a V sign [2]. The thalamus has 4 regions of vascular supply which are known as the anterior, paramedian, inferolateral, and posterior regions. The vascular supply of the bilateral medial thalami and rostral midbrain is supplied by the paramedian arteries [5]. The artery of Percheron is a variant in which there is a single arterial supply to both paramedian thalami. Therefore, an occlusion in the AOP results in loss of both paramedian nuclei. Unilateral paramedian infarct presents with impaired arousal, confusion, apathy, and memory impairment in areas of speech and language production; hypophonia and dysprosdy [5].

A bilateral paramedian infarct presents with hypersomnolence, comatosis, mutism, and abnormal eye movements [1]. It is comparative to the lock-in component of a top of the basilar artery infarct. Recovery from a thalamic infarct is generally regarded as having a better prognosis than from a cerebral or another subcortical area of infarct in all age groups [5]. Nonetheless, psychiatric and cognitive deficits are known to persist and depend on the severity of the AMS on initial presentation; the size of the infarct, and whether there is involvement of the ventricular system or not [5]. AOP is too small to be seen on CTA or MRA, and MRI-DWI is the preferred method of diagnosis [6].

\section{Discussion}

The change in mental status was first noticed at the nursing home by the patient's companion upon trying to feed a patient who did not swallow or respond. The family reported that at baseline patient was minimally ambulatory with a wheelchair, capable of self-feeding; and produced non-communicative vocalizations. She notified the nursing home staff; however, the patient was not sent to the hospital until the following day; so the duration from the first recognition of symptoms to the ICU was at least over 48 hours, and it excludes the time from the actual first onset of symptoms at the nursing home. Difficulty in early awareness was compounded by the patient's significant baseline comorbidities; advanced Alzheimer's dementia, bilateral sensorineural deafness, and enucleation of the left eye with cataracts in the right; making vertical gaze palsy difficult to recognize.

At first, there was a concern that the patient's encephalopathy was due to acute hypercapnic respiratory failure secondary to the viral illness/aspiration pneumonia. However, 
this could not explain the extent of his comatosis as he was maintaining adequate oxygen saturation on room air, afebrile without leukocytosis, negative blood cultures and clear CXR; prompting further investigation with MRI.

The patient's family who was now by bedside wanted to know a clear etiology of the cause of his change in mental state given that the above workup was negative. It was explained to the family that at this point the outcome in management would likely not change.

Given patient's known history of atrial fibrillation; not on anticoagulation; the acute onset of symptoms together with MRI consistent with stroke and a known cardio-embolic source; etiology of the comatose state became clear. In this case, the patient was DNR/DNI; and refused feeding tubes on prior documentation; and thus family opted to take the patient to the nursing home on comfort care measures.

Although in this case earlier recognition may not have improved outcomes in morbidity as this patient likely presented at least 48 hours after the behavioral change was first reported, early recognition is essential so that thrombolysis and appropriate therapy could be administered in the window of treatment that could drastically change outcomes; with delay leading to higher morbidity and mortality. An AOP infarct should be suspected as a cause of stroke in young patients that present with coma, hypersomnolence and vertical gaze paralysis; in the absence of other risk factors and a diffusion-weighted MRI is the preferred diagnostic test.

\section{Conclusion}

High suspicion of bilateral thalamic stroke should be maintained in a patient that presents with altered mentation; hyper-somnolence or comatosis, and ophthalmologic signs like vertical gaze palsy in the presence of pre-existing cardioembolic risk factors. MRI must be performed as it is the modality of choice to detect this type of stroke.

\section{References}

1. Caruso P, manganotti P, Moretti R (2016) Complex neurological symptoms in bilateral thalamic stroke due to Percheron artery occlusion. Vasc Health and Risk Mgmt13:11-14.

2. Jumean KA, Arqoub A, Al Hadidi MA, Hawatmeh A, Shaaban H (2016) Bilateral thalamic stroke due to occlusion of the artery of Percheron in a patient with a patent foramen ovale. Journal of Natural Science, Biology and Medicine 7:109-112.

3. Lazzaro NA1, Wright B, Castillo M, Fischbein NJ, et al. (2010) Artery of Percheron Infarction: Imaging Patterns and Clinical Spectrum. American Journal of Neuroradiology 31:1283:1289.

4. Schmahmann JD (2003) Vascular Syndromes of the thalamus 34:2264-2278.

5. Singh P, Kaur R, Kumar N (2016) Ischemic stroke due to occlusion of the artery of Percheron. Indian Journal of Critical Care Medicine 20:251-252.

6. Muhammad A, Appu S, Syed A, Sonni JSK (2015) A case of the midbrain and thalamic infarction involving the artery of Percheron. Journal of Clinical Medicine 4:369- 374.

Submit your manuscript to a JScholar journal and benefit from:

- Convenient online submission

ब Rigorous peer review

I Immediate publication on acceptance

ब Open access: articles freely available online

q High visibility within the field

- Better discount for your subsequent articles

Submit your manuscript at http://www.jscholaronline.org/submit-manuscript.php 